\title{
A CROSS-SECTIONAL STUDY TO EVALUATE THE USEFULNESS OF 'PRESSURE TO CORNEA INDEX' IN PREDICTION OF PRIMARY OPEN ANGLE GLAUCOMA
}

\author{
Pandiyaraj Saravanasankar ${ }^{1}$, Anbazhaghan Amudha², Srinivasan Muralikrishnan ${ }^{3}$, Reethysreekumar Sruthi ${ }^{4}$, Rajan Saranya ${ }^{5}$, \\ Muthusamy Charanya ${ }^{6}$, Jothimani Deebalakshmi ${ }^{7}$
}

\begin{abstract}
${ }^{1}$ Assistant Professor, Department of Ophthalmology, Madurai Medical College. ${ }^{2}$ Assistant Professor, Department of Ophthalmology, Madurai Medical College. 3Junior Resident, Department of Ophthalmology, Madurai Medical College. 4Junior Resident, Department of Ophthalmology, Madurai Medical College. 5Junior Resident, Department of Ophthalmology, Madurai Medical College. 6Junior Resident, Department of Ophthalmology, Madurai Medical College. 7Junior Resident, Department of Ophthalmology, Madurai Medical College.
\end{abstract}

\section{BACKGROUND}

ABSTRACT

The prevalence of glaucoma blindness is about $8 \%$ of global blindness and in India is about 4.4\%. The Intra ocular Pressure (IOP) is a single major modifiable risk factor in glaucoma. In order to integrate IOP and CCT as a single risk factor, a new index 'Pressure-toCornea Index' (PCI) was proposed. But studies regarding the fact that the increased values of PCI will be a risk factor for glaucoma are limited. This study attempts to describe the role of PCI as a predictive risk factor in clinical POAG.

The aims and objectives of this study are to determine the PCI values in POAG, OHT and NTG and to determine whether PCI can be used as a predictive value in clinical Primary Open Angle Glaucoma (POAG).

\section{MATERIALS AND METHODS}

This observational, cross-sectional type of study was conducted over a period of six months, in 478 eyes of patients attending the outpatient at Govt. Rajaji Hospital, Madurai, who satisfied the inclusion-exclusion criteria. IOP estimation by GAT and CCT measurement by Ultrasound Pachymetry was done in all of them. After routine glaucoma evaluation, they were segregated as Group 1: Normotensives (374), Group 2: Ocular Hypertension (29), Group 3: Normal Tension Glaucoma (28) and Group 4: Primary Open Angle Glaucoma (47). Data collected were entered in Master Chart and statistical analysis was done using software IBM SSPS Ver 21.0 (Armonk, NY) by our statistician. The study groups without clinical POAG are Group 1 and Group 2 (mean PCI value of 92 and 117). The study groups with clinical POAG are Group 3 and Group 4 (mean PCI value of 134 and 171). This study demonstrates a significant correlation between PCI levels and glaucomatous changes in eyes. The PCI value may be also useful in the management of glaucoma for setting a target IOP.

\section{RESULTS}

The obtained ' $\mathrm{P}$ ' value is $<0.001$ which is statistically significant says that there is a difference in the IOP measurements and CCT measurements between the 4 groups (Normal, Ocular Hypertension, Normotensive Glaucoma and Primary Open Angle Glaucoma).

\section{CONCLUSION}

The value of PCI is significantly increased in glaucomatous (POAG and NTG) eyes. This emphasises that PCI can be used as a predictive index in development of glaucomatic changes in optic disc in the population.

\section{KEYWORDS}

Corneal Thickness Measurement, Open Angle Glaucoma, Intraocular Pressure.

HOW TO CITE THIS ARTICLE: Saravanasankar P, Amudha A, Muralikrishnan S, et al. A cross-sectional study to evaluate the usefulness of 'pressure to cornea index' in prediction of primary open angle glaucoma. J. Evolution Med. Dent. Sci. 2018;7(01):4749, DOI: $10.14260 /$ jemds/2018/12

\section{BACKGROUND}

Glaucoma is a chronic progressive disease with a triad of increased Intraocular Pressure (IOP) and progressive optic neuropathy, both resulting in specific pattern of irreversible Visual Field Defects (VFD).

'Financial or Other Competing Interest': None.

Submission 07-11-2017, Peer Review 14-12-2017,

Acceptance 20-12-2017, Published 01-01-2018.

Corresponding Author:

Dr. Pandiyaraj Saravanasankar,

Assistant Professor of Ophthalmology,

Eye Department OP No.50,

Government Rajaji Hospital,

No. 1, Panagal Road, Madurai-625020, Tamilnadu.

E-mail: eyedrck@gmail.com

DOI: $10.14260 /$ jemds $/ 2018 / 12$
The prevalence of glaucoma blindness is about $8 \%$ of global blindness ${ }^{1}$ and in India is about $4.4 \% .^{2}$ Several large multicentre randomised clinical trials confirmed the role of Intraocular Pressure (IOP) as a single major modifiable risk factor in glaucoma. ${ }^{3}$

The level of IOP is usually over $21.0 \mathrm{mmHg}$, where clinical Primary Open Angle Glaucoma (POAG) is seen. The normal Central Corneal Thickness (CCT) ranges from $490 \mu-560 \mu$. But many studies have pointed out that the CCT affects the accuracy of IOP measurement using Applanation methods. ${ }^{4}$ With Goldmann Applanation Tonometer (GAT), there was an overestimation of IOP in thick corneas and underestimation of IOP in thin corneas. ${ }^{5}$ Ultrasound pachymetry is used for estimating the CCT. In an attempt to correct the applanation IOP according to CCT values, several conversion tables and 
formulas have been suggested.6,7 However, there is a wide disagreement among investigators.

In order to integrate IOP and CCT as a single risk factor, a new index 'Pressure-to-Cornea Index' (PCI) was proposed. ${ }^{8}$ The authors believed that PCI could better reflect the individual's susceptibility to glaucomatous damage than either of the individual parameters, IOP/ CCT alone. ${ }^{9}$ But studies regarding the fact that the increased values of PCI will be a risk factor for glaucoma are limited. This study attempts to describe the role of $\mathrm{PCI}$ as a predictive risk factor in clinical POAG.

\section{Aims and Objectives}

1. To determine the PCI values in POAG, OHT and NTG.

2. To determine whether PCI can be used as a predictive value in clinical Primary Open Angle Glaucoma (POAG).

\section{MATERIALS AND METHODS}

This observational, cross-sectional type of study was conducted over a period of six months in 478 eyes of 252 patients attending the outpatient Department of Ophthalmology, Govt. Rajaji Hospital, Madurai, who satisfied the inclusion-exclusion criteria. The formula used by our statistician to determine this sample size was $\left[\mathrm{Z}_{1-\alpha / 2}{ }^{2} \mathrm{p}(1-\mathrm{p})\right] /$ $d^{2}$, where $Z_{1-\alpha / 2}$ is the standard normal variate at $5 \%$ type $I$ error $(\mathrm{p}<0.05)$ which is 1.96 , ' $\mathrm{p}$ ' is the expected proportion in population based on pilot study of $15 \%$ and ' $d$ ' is the absolute error or precision of $5 \%$. Patients in the age group of 40 - 70 years of either sex following the inclusion-exclusion criteria and who consented were included in this study. Persons with anatomically primary narrow anterior chamber angle, secondary glaucoma, corneal disorders, refractive corneal surgeries, contact lens wear at the time of CCT measurement, intraocular disorders other than mild cataract, myopia - greater than 6.0 Diopter, hyperopia - greater than 4.0 Diopter, astigmatism greater than 2 Diopter and patients not consenting for this study were excluded from our study.

IOP estimation by GAT and CCT measurement by Ultrasound Pachymetry was done in all of them. After routine glaucoma evaluation they were segregated as Group 1: Normotensives- No clinical POAG and Untreated IOP $<$ or equal to $21 \mathrm{mmHg}$; Group 2: Ocular Hypertension (OHT) - No clinical POAG, but untreated IOP > $21 \mathrm{mmHg}$; Group 3:
Normal Tension Glaucoma (NTG) - Established glaucomatous optic disc and visual field damage, untreated IOP < or equal to $21 \mathrm{mmHg}$; and Group 4: Primary Open Angle Glaucoma (POAG) - Established glaucomatous optic disc and visual field damage, untreated IOP > $21 \mathrm{mmHg}$. The Pressure-to-Cornea Index (PCI) was calculated as the ratio between initial presenting IOP and cubic power of CCT expressed in $\mathrm{mm}$ for each subject $\mathrm{PCI}=\mathrm{IOP}$ in $\mathrm{mmHg} /(\mathrm{CCT}$ in $\mathrm{mm}) .{ }^{3}$ Data collected were entered in Master Chart and statistical analysis was done using software IBM SSPS Ver 21.0 (Armonk, NY).

\section{RESULTS}

Four hundred and seventy eight (478) eyes were used in the study, of which there was clinical POAG in 47 eyes (Group 1); NTG in 28 eyes (Group 2) and OHT in 29 eyes (Group 3). The remaining 374 eyes had no clinical POAG (Group 4). The IOP and CCT were measured and the values were entered in the Master Chart. The PCI was calculated for each of the study eyes. The distribution of the mean, standard deviation and range of IOP, CCT and PCI in the four groups was determined; Table 1 . The continuous variables were given with mean (standard deviation) and categorical variables were presented with frequency (percentage). The normality of the data was checked using Shapiro-Wilk test. The parameters such as IOP, CCT and PCI were compared between the different groups using a statistical test called \#one-way ANOVA test. $P$ value $<0.05$ were considered as statistically significant. All the statistical analysis was done using statistical software IBM SPSS Ver 21.0 (Armonk, NY). The obtained ' $\mathrm{P}$ ' value of $<0.001$, which is statistically significant says that there is a difference in the IOP and CCT measurements and PCI values between the 4 groups (Normal, Ocular Hypertension, Normotensive Glaucoma and Primary Open Angle Glaucoma). A post-hoc test called \#\#Tukey's test was used for the pairwise comparison of each parameter. It was found that only normotensive glaucoma vs. normal $(\mathrm{P}=0.156)$ and POAG Vs. Ocular hypertension $(\mathrm{P}=0.444)$ for IOP was not statistically significant $(P>0.05)$. Thus, it is clear that IOP alone is not a reliable parameter in differentiating Normotensive glaucoma and Ocular hypertension; Table 2. As both the CCT and PCI values show statistically significant difference between all the groups, these parameters prove to be better and more reliable indicators of clinical glaucoma.

\begin{tabular}{|c|c|c|c|c|c|c|}
\hline \multicolumn{2}{|c|}{ Parameter } & Group $1(n=374)$ & Group $2(n=28)$ & Group $3(n=29)$ & Group $4(n=47)$ & P value $\#$ \\
\hline \multirow{2}{*}{ IOP } & Mean (SD) & $15.84(2.88)$ & $24.35(1.96)$ & $17(2.6)$ & $26.36(2.85)$ & \multirow{2}{*}{$<0.001$} \\
\hline & Range & $8-22$ & $22-29$ & $12-21$ & $23-36$ & \\
\hline \multirow{2}{*}{ CCT } & Mean (SD) & 549.1 (14.11) & $592.42(6.99)$ & $502.24(9.83)$ & 536.13 (14.27) & \multirow{2}{*}{$<0.001$} \\
\hline & Range & $501-578$ & $578-612$ & $485-525$ & $505-560$ & \\
\hline \multirow[b]{2}{*}{ PCI } & Mean (SD) & $97.68(11.14)$ & $117.42(7.69)$ & $134.27(8.36)$ & $171.04(12.95)$ & \multirow{2}{*}{$<0.001$} \\
\hline & Range & $68-178$ & $105-139$ & $125-156$ & $153-193$ & \\
\hline
\end{tabular}

\begin{tabular}{|c|c|c|c|}
\hline Pairwise Comparison\#\# & IOP P Value & CCT P Value $^{\#}$ P Value \\
\hline $\begin{array}{c}\text { Ocular Hypertension } \\
\text { Vs. Normal }\end{array}$ & $<0.001$ & $<0.001$ & $<0.001$ \\
\hline Normotensive Glaucoma Vs. Normal & 0.146 & $<0.001$ & $<0.001$ \\
\hline POAG Vs. Normal & $<0.001$ & $<0.001$ & $<0.001$ \\
\hline Normotensive Glaucoma Vs. Ocular Hypertension & $<0.001$ & $<0.001$ & $<0.001$ \\
\hline POAG Vs. Ocular Hypertension & 0.444 & $<0.001$ & $<0.001$ \\
\hline POAG Vs. Normotensive Glaucoma & $<0.001$ & $<0.001$ & $<0.001$ \\
\hline \multicolumn{2}{|c|}{} \\
\hline
\end{tabular}




\section{DISCUSSION}

The corneal thickness is said to influence the measurement of IOP, thereby influencing the glaucoma diagnosis. This makes IOP sometimes unreliable in a few subjects who have the same features of POAG except for increased IOP. This study evaluates the new index PCI for predicting glaucoma, proposed by Iliev et al. The study groups with clinical POAG are Group 3 and Group 4 (mean PCI value of 134 and 171). The study groups without clinical POAG are Group 1 and Group 2 (mean PCI value of 92 and 117). The mean value of PCI in the study groups is more or less consistent with a previous study done by Iliev et al. The average CCT in normal population study group was calculated to be $549.1 \mu \mathrm{m}$. It was found that NTG eyes had thinner than average and OHT eyes had thicker corneas than average corneal thickness. For IOP values $\geq 22$, the cut-off value of PCI given by Iliev et al in his study was 140 and for values $\leq 21$, the cut-off value of PCI was 120 . This study holds a good concordance with this since $76.6 \%$ of POAG eyes had PCI value above 140 and $65.29 \%$ of NTG eyes had PCI above 120; $86.20 \%$ of OHT had PCI value below 140 and $89.57 \%$ of normal population had PCI below 120. This study demonstrates a significant correlation between PCI levels and glaucomatous changes in eyes.

PCI was found to differentiate glaucomatous from nonglaucomatous eyes better when compared to corrected IOP to CCT. The PCI value which best differentiated glaucoma and non-glaucoma was found to be 117 in this study, which when compared with the study done by Iliev et al was 133.8. It is very clear that PCI value below 120 belongs to the domain of normals and above that belongs to the glaucoma. PCI can be used as an additional parameter in context of other clinical findings in routine investigations like outflow facility, thus facilitating in earlier diagnosis and reducing the morbidity. Thus, a low PCI indicates low risk of glaucomatous damage and vice versa. The PCI value may be also useful in the management of glaucoma for setting a target IOP. So further long-term studies are needed to prove the prognostic value of PCI and how it correlates to the pathogenesis of glaucoma.

\section{CONCLUSION}

The value of PCI is significantly increased in glaucomatous (POAG and NTG) eyes. This emphasises that PCI can be used as a predictive index in development of glaucomatic changes in optic disc in the population.

\section{Limitations of this Study}

The limitation of this study was that the number of subjects included in the study was minimal. So long-term studies involving large scale population are essential to support the reliability of PCI in predicting the risk of glaucoma.

\section{REFERENCES}

[1] The AGIS Investigators. The Advanced Glaucoma Intervention Study (AGIS): 7. The relationship between control of intraocular pressure and visual field deterioration. AM Ophthalmol 2000;130(4):429-40.

[2] Shimmyo M, Ross AJ, Moy A, et al. Intraocular pressure, Goldmann applanation tension, corneal thickness and corneal curvature in Caucasians, Asians, Hispanics and African Americans. Am J Ophthalmol 2003;136(4):603-13.

[3] Leske MC, Heijl A, Hussein M, et al. Factors for glaucoma progression and the effect of treatment: the early manifest glaucoma trial. Arch Ophthalmol 2003;121(1):48-56.

[4] Thomas R, Korah S, Muliyil J. The role of central corneal thickness in the diagnosis of glaucoma. Indian J Ophthalmol 2000;48(2):107-11.

[5] Shah S, Chatterjee A, Mathai M, et al. Relationship between corneal thickness and measured intraocular pressure in a general ophthalmology clinic. Ophthalmology 1999;106(11):2154-60.

[6] Ehlers N, Bramsen T, Sperling S. Applanation tonometry and central corneal thickness. Acta Ophthalmol (Copenh) 1975;53(1):34-43.

[7] Matsumoto T, Makino H, Uozato H, et al. The influence of corneal thickness and curvature on the difference between IOP measurements obtained with a non-contact tonometer and those with a Goldmann applanation tonometer. Nippon Ganka Gakkai Zasshi 2000;104(5)317-23.

[8] Illiev ME, Meyenberg A, Buerki E, et al. Novel pressure-to-cornea index in glaucoma. $\mathrm{Br} \mathrm{J}$ Ophthalmol 2007;91(10):1364-8.

[9] Ko YC, Liu CJ, Hsu WM. Varying effects of corneal thickness on intraocular pressure measurements with different tonometers. Eye (Lond) 2005;19(3):327-32. 